\title{
Survey on Image Watermarking Schemes using Adaptive Soft Computing Techniques
}

\author{
Amita Goel \\ Department of Computer Science, \\ Teerthanker Mahaveer University \\ Moradabad,India
}

\author{
Anurag Mishra \\ Department of Electronics, \\ Deendayal Upadhyay College, University of Delhi, \\ New Delhi,India
}

\begin{abstract}
During last few years, many soft computing techniques have been employed for image watermarking. These are more into delving the issue of optimization of visual quality of signed images and robustness of the embedding algorithm. The used techniques either operate in adaptive or learning mode, especially those using Artificial Neural Networks or in non adaptive analytical mode such as ones based on Fuzzy logic. Several researchers have also worked on this problem using hybrid and evolutionary algorithms. This research survey especially deals with the image watermarking techniques which rely on adaptive soft computing techniques. The results of gradient descent based Back propagation Network (BPN algorithm, Radial Basis Function Neural Network (RBFNN algorithm and a newly developed Single Layer Feed forward Neural Network (SLFN algorithm commonly known as Extreme Learning Machine (ELM) used to carry out watermarking in uncompressed grayscale images are compared. These techniques are compared for different images and the comparison is based on the visual quality of signed images, the watermark detector response coefficients such as similarity correlation and normalized correlation parameters and the robustness studies. Time complexity issue is also examined to establish the use of watermarking process on a real time scale. It is concluded that the ELM algorithm gives a reasonable generalized behavior in terms of computation of these parameters as compared to its other counterparts. It's fast training in milliseconds and subsequent embedding and extraction makes it suitable for developing watermarking application on a real time scale.
\end{abstract}

\section{General Terms}

Image Watermarking, Soft Computing Techniques

\section{Keywords}

BPN, Radial Basis Function Neural Network, ELM

\section{INTRODUCTION}

Watermarking of grayscale images is an advanced area of research in the signal processing domain. It is frequently used for copyright protection, owner identification and content authentication and authorization. The content authentication and authorization specifically helps consumers to:

- Find how, when and where the copyrighted content is being consumed

- Protect the digital content from any possible unauthorized use

- Link consumers to and from the content that is relevant to their interests

- Provide a way for users to easily identify the source of content by communicating copyright information and identify the appropriate channels for licensing or purchasing

- Organize and manage content in a digital asset management system

- Identify the source of leaks when confidential content inadvertently or intentionally makes its way onto the Internet

Figure 1 depicts a typical image watermarking system [15].

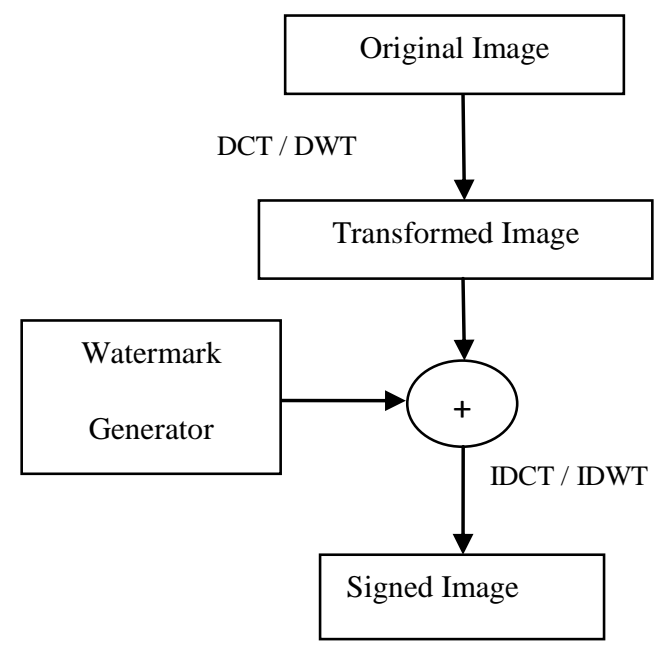

Figure 1: A typical image watermarking system

To this end, several robust image watermarking schemes have been developed [1-4]. Broadly, these schemes belong to pure signal processing domain in which the algorithms specifically deal with pixel coefficients in transform domain using standard mathematical formulations. Another branch of algorithmic evolution deals with the development of watermarking schemes using standard soft computing techniques. This is particularly due to the fact that the problem of watermarking of images is now converged as an optimization problem. The twin parameters - visual quality of the signed images and the robustness of the embedding scheme are often found to be mutually exclusive, keeping the embedding capacity as constant. This is more because, the embedded content is far less than the size of the host signal (grayscale image). If visual quality is enhanced, it is often carried out at the cost of robustness which makes the signed images vulnerable to signal processing attacks, thereby easily removing the watermark. On the other hand, if robustness is increased beyond a certain limit, it can only be done at the cost of visual quality of the signed images which makes the signed content hardly of any practical use. To optimize these parameters, adaptive soft computing techniques are 
extensively being used worldwide. These techniques mainly Different architectures. Gradient Descent based Back Propagation Neural Network (BPN) and Radial basis Function Neural Network (RBFNN) algorithms are frequently used for this purpose. These network architectures are indeed very good at learning and therefore present an efficient solution to the optimization of the aforesaid parameters. However, BPN often gets trapped in the local minima and seldom finds global optimum solutions efficiently. This makes it a costly candidate to implement image watermarking in terms of time.

Radial basis function Neural Network is comparatively better in terms of time. However, overall processing time requirement is very high in this case. It has been observed that till now, a comprehensive comparative study on adaptive soft computing techniques utilized for the purpose of image watermarking is lacking. This brief survey presents an introductory approach in this direction and is limited to neural network based individual and hybrid strategies to carry out watermarking in grayscale host images. This survey focuses on the overall outcomes of these watermarking schemes in terms of visual quality of signed and attacked images and robustness studies as well as the processing time spans utilized to implement various processes involved. A comparison shall be made on the basis of the time spans especially to highlight its possible usage for implementing watermarking on a real time scale meeting strict timelines.

\section{RELATED RESEARCH WORK}

Huang et al. [5] developed a novel blind watermarking technique based on Back Propagation Neural Network in the wavelet domain. According to this scheme, with the use of HVS characteristics, a scrambled watermark is imperceptibly embedded in a robust manner. A neural network is successfully used to memorize the relation between the watermark and the corresponding watermarked image. This way, the authors have been successful to blindly recover the exact watermark from the signed image. Their results indicate

that the proposed scheme offers good imperceptibility and high robustness to various image processing attacks.

Chang et al. [6] have proposed a novel method known as Full Counter-Propagation Neural Network (FCNN) for implementing digital image watermarking. In this watermarking scheme, the watermark is embedded and extracted through specific FCNN. This adaptive method is different from the traditional methods as in this case the multiple cover images and the watermark are embedded in the synapses of a FCNN simultaneously instead of the cover images. Therefore, the watermarked image is almost the same as the original cover image. In addition, most of the attacks could not degrade the quality of the extracted watermark image. The experimental results show that the proposed method is able to achieve robustness, imperceptibility and authenticity in watermarking. The authors have been able to achieve good visual quality of the signed and attacked images and high normalized correlation value indicates good robustness of the embedding scheme. The authors, however, do not compute any processing time spans consumed by their algorithm.

Ri Piao et al. [7] proposed a new Human Visual System (HVS) model based blind watermarking scheme in which a watermark is embedded into the discrete wavelet transform (DWT) domain. This method uses the HVS model and Radial Basis Function Neural Network (RBFNN). They use RBFNN to embed and extract the watermark. They further use the involve Artificial Neural Networks having HVS characteristics to determine the insertion strength of the random sequence watermark. A secret key is used to determine the beginning position of the image where the watermark is to be embedded. The authors claim that this process prevents pirates from removing the watermark easily. Their experimental results show that their method has good imperceptibility and high robustness to the common image processing attacks. They report that they have obtained a PSNR value greater than $45 \mathrm{~dB}$ in case of all images. They claim that due to adaptive capabilities of the RBF network, the embedding / extraction strategy can generally improve robustness against the selected image processing attacks. They do not compute the processing time spans of RBF network training, embedding and extraction.

Zhang Zhi-Ming et al. [8] propose an adaptive digital watermarking scheme with RBF neural networks, in which a visually recognizable binary image watermark is embedded into the DCT domain of the cover image. The watermark was encrypted by chaotic series and inserted into the middle frequency coefficients of the cover image's blocked DCTbased transform domain. According to authors, to make the watermark stronger to resist different types of attacks, it is important to adapt the embedding maximum amount of interested watermark before the watermark becomes visible. In this paper, RBF neural networks are used to achieve maximum-strength watermark according to the frequency component feature of the cover image. Experimental results show that the proposed techniques have good imperceptibility and can survive of common image processing operations and JPEG lossy compression with high robustness. They do not compute processing time spans of any process utilized in this algorithm.

Ali Mohammad Latif [9] proposed an adaptive hybrid approach to carry out image watermarking. This is based on fuzzy logic and an evolutionary computing technique known as tabu search. This scheme also makes use of HVS model. They use Hadamard transform to transfer the image from spatial domain to transform domain. The selected transform includes parameters that can be handled to control the requirements of watermarking such as robustness and the visual quality of signed images. They apply the transform parameters to enhance the robustness by tabu search and after having carried out the embedding process, the watermark is adapted to the image by exploiting HVS characteristics using fuzzy gradient to ensure good visual quality. They claim that their experimental results behave optimally and show high imperceptibility as well as high robustness against variety of attacks.

Agarwal et al. [10] have proposed a novel watermarking scheme by embedding a binary watermark into four different grayscale images using a hybrid GA-BPN intelligent network. In this paper, HVS characteristics of the host images in DCT transform domain are used to obtain a sequence of weighting factor from a GA-BPN. This weighting factor is used to embed and extract the watermark from the image in DWT domain. The GA-BPN is trained by 27 inference rules that includes three input HVS parameters namely luminance sensitivity, edge sensitivity computed using threshold and contrast sensitivity computed using variance. The robustness of the embedding scheme is examined by executing seven different image processing attacks. Visual quality of signed images before and after the attacks is examined by PSNR. The extracted watermarks from signed and attacked images show a high degree of similarity with the embedded content. The 
authors claim that the algorithm is robust against selected attacks and is well optimized. The normalized correlation parameter is very high thereby indicating that the watermark recovery is quite efficient. In other words, the proposed algorithm is robust against the seven selected attacks. They have also computed the training time of the used GA-BPN network as well as the embedding and extraction time spans. In addition, the time spans for executing image processing attacks is also computed by them. They report that in case of all four images, the GA-BPN based algorithm consumes about 14 - 16 seconds / image to carry out embedding and extraction from different signed and attacked images. Although, the reported time span for an individual image is less, however on a large scale, especially in case of a large video, the embedding and extraction may consume much more time.

All these algorithms are used to implement image watermarking and its related issues such as minimizing tradeoff between visual quality of the signed images and the robustness. However, there is very less work done to compute processing time for embedding and extraction of watermark. Therefore, it remains an interesting avenue to examine the embedding and extraction processing time span to develop a faster watermarking scheme capable to complete the task on a real time scale. This may further be extended for video also. E. G. B. Huang et al. have developed a fast algorithm for training of ANNs with a completely different architecture in place, popularly known as the extreme learning machine (ELM). The benefits of this approach are that it has only one tunable parameter, namely, the number of hidden neurons, and its training process consists of only a single step, thereby reducing the training time up to a large extent. The training of this machine is found to be extremely fast and on regular image databases used by authors, it is reported to have been finished within milliseconds with a reasonably good accuracy [11-13].

Mishra et al. [14] have recently proposed a novel digital image watermarking algorithm based on Extreme Learning Machine (ELM) for two gray scale images. As mentioned, the ELM algorithm is very fast and completes its training in milliseconds unlike its other counterparts such as BPN or Fuzzy Inference System (FIS). In this paper, they report that their algorithm trains the ELM by using low frequency coefficients of the grayscale host image in transform domain. The trained ELM produces a sequence of 1024 real numbers, normalized according to $\mathrm{N}(0,1)$ as an output. They use this sequence as the watermark to be embedded within the host image using Cox's formula [15] to obtain the signed image. The visual quality of the signed images is quantified by PSNR. The authors report high PSNR values which indicate that the quality of signed images is good. The computed high value of SIM $\left(\mathrm{X}, \mathrm{X}^{*}\right)$ establishes that the extraction process is successful and according to authors, the algorithm finds good practical applications, especially in situations that warrant meeting time constraints. This is because, they obtain the training time

Very recently, Singh et al. have demonstrated the use of ELM to carry out watermark embedding, extraction and robustness studies in colored bitmap images in DWT domain [16]. In this paper, the authors claim to have exhibited the efficacy of Extreme Learning Machine (ELM) algorithm for watermarking of images in Discrete Wavelet Transform domain. According to the authors, ELM is a regularization algorithm which works on the concept of generalized singlehidden-layer feed forward neural networks (SLFNs) with different activation functions such as RBF, SINE, SIGMOID and HARDLIM in hidden nodes in unified environment framework. In this learning method, the parameters of hidden nodes like the input weight and bias value of the additive nodes are randomly selected based on input data samples. This algorithm is developed for batch learning and show good generalization performance. Except from selecting the number of hidden nodes, no other learning parameter is adopted here manually. The authors test detail performance and efficacy of this algorithm to implement watermarking of color images in Discrete Wavelet Transform (DWT) domain. Their results show that watermarking scheme based on ELM is robust and imperceptible. It also gives better generalization performance against common image processing attacks. However, they do not compare their results with any of the previously reported adaptive strategies to carry out watermark embedding and extraction.

Table 1. PSNR values of Lena and Baboon images by different watermarking techniques

\begin{tabular}{|c|c|c|c|c|}
\hline \multicolumn{2}{|c|}{$\begin{array}{l}\text { Watermarking } \\
\text { Technique }\end{array}$} & \multirow{3}{*}{$\begin{array}{c}\begin{array}{c}\text { Publish } \\
\text { ed } \\
\text { Work }\end{array} \\
\begin{array}{c}\text { Isac et } \\
\text { al.[18] }\end{array}\end{array}$} & \multirow{2}{*}{$\begin{array}{c}\begin{array}{c}\text { PSNR } \\
(\text { dB })\end{array} \\
\text { Lena }\end{array}$} & \multirow{2}{*}{\begin{tabular}{|c}
$\begin{array}{c}\text { PSNR } \\
\text { (dB) } \\
\text { Baboon }\end{array}$ \\
28.84
\end{tabular}} \\
\hline \multirow{5}{*}{$\begin{array}{c}\text { Neural } \\
\text { Network }\end{array}$} & $\mathrm{CNN}$ & & & \\
\hline & BPN & & 22.51 & 35.68 \\
\hline & $\mathrm{RBF}$ & & 38.68 & 38.27 \\
\hline & Hopfield & & 34.45 & 36.25 \\
\hline & FCNN & & 37.49 & 48.68 \\
\hline \multirow{5}{*}{$\begin{array}{l}\text { Fuzzy } \\
\text { Logic } \\
\text { and Tabu } \\
\text { Search }\end{array}$} & $\begin{array}{c}\text { Classical } \\
\text { Slant }\end{array}$ & \multirow[t]{5}{*}{$\begin{array}{c}\text { Latif et } \\
\text { al. [9] }\end{array}$} & 50.31 & 50.81 \\
\hline & $\begin{array}{l}\text { Walsh- } \\
\text { Hadamar } \\
\text { d }\end{array}$ & & 48.95 & 49.19 \\
\hline & $\begin{array}{l}\text { Constant } \\
\text {-Betas }\end{array}$ & & 52.75 & 51.24 \\
\hline & $\begin{array}{l}\text { Multiple- } \\
\text { Betas }\end{array}$ & & 53.32 & 52.18 \\
\hline & $\begin{array}{c}\text { DCT } \\
\text { Domain }\end{array}$ & & 43.04 & 43.05 \\
\hline \multicolumn{2}{|c|}{$\begin{array}{l}\text { GA-BPN Hybrid } \\
\text { Network }\end{array}$} & $\begin{array}{c}\text { Agarwa } \\
\text { l et } \\
\text { al.[10] }\end{array}$ & 37.63 & 39.53 \\
\hline \multirow{2}{*}{\multicolumn{2}{|c|}{$\begin{array}{l}\text { Support Vector } \\
\text { Machine }\end{array}$}} & $\begin{array}{l}\text { Yu et. } \\
\text { al.[19] }\end{array}$ & 43.57 & 43.62 \\
\hline & & $\begin{array}{l}\text { Yen et } \\
\text { al.[17 ] }\end{array}$ & 45.53 & 43.06 \\
\hline \multicolumn{2}{|c|}{$\begin{array}{l}\text { Extreme Leaning } \\
\text { Machine using DCT }\end{array}$} & $\begin{array}{c}\text { Mishra } \\
\text { et } \\
\text { al. }[14]\end{array}$ & 60.40 & 62.80 \\
\hline
\end{tabular}


Table 1 compiles computed values of PSNR for Lena and Baboon images. Several adaptive neural network techniques such as CNN, BPN, RBF, Hopfield and FCNN are used to carry out watermarking and their results indicate that for these methods, the PSNR value is less in comparison to the PSNR obtained using the fast ELM based watermarking technique. Similarly, the GA-BPN Hybrid network and Support Vector Regression (SVR) based methods also give smaller PSNR values for Lena and Baboon. The high value of computed PSNR value for Lena and Baboon using ELM technique indicates that the visual quality of the signed image is quite good in comparison to the other soft computing techniques. Latif et al. [9] computed BCR $=100$ for Fuzzy logic and Tabu Search based watermarking scheme and Agarwal et al. [10] reported NC $=1.00$ for GA-BPN based hybrid watermarking scheme for Lena and Baboon images respectively. Similarly, Chang et al. [7] reported NC $=0.996$ for the Baboon image. Table 2 shows the SIM values computed for signed images obtained by using GA-BPN [10] and ELM [14] based schemes.

Table 2. SIM values for Lena and Baboon Image for different watermarking techniques

\begin{tabular}{|c|c|c|}
\hline \multirow{2}{*}{$\begin{array}{l}\text { Published } \\
\text { Work }\end{array}$} & \multicolumn{2}{|c|}{ SIM } \\
\cline { 2 - 3 } & (Lena) & (Baboon) \\
\hline $\begin{array}{c}\text { Agarwal } \\
\text { et al. [10] }\end{array}$ & 15.2470 & 15.2422 \\
\hline $\begin{array}{c}\text { Mishra et } \\
\text { al. [14] }\end{array}$ & 20.4956 & 26.3466 \\
\hline
\end{tabular}

It is quite clear that the ELM based watermarking scheme is able to outperform GA-BPN based hybrid watermarking scheme for watermark extraction. Table 3 summarizes the embedding and extraction time of watermarking process by using several techniques up to the extent that they have reported the same. Note that most of these works do not present their embedding and extraction time spans. From this summarized data, it is surely concluded that the embedding and extraction by using Extreme Learning Machine is very fast.

Table 3 summarizes the embedding and extraction time of watermarking process by using several techniques up to the extent that they have reported the same. Note that most of these works do not present their embedding and extraction time spans. From this summarized data, it is surely concluded that the embedding and extraction by using Extreme Learning Machine is very fast.

Table 3. Embedding and Extraction time spans for Lena and Baboon by different watermarking techniques

\begin{tabular}{|c|c|c|c|c|}
\hline & $\begin{array}{c}\text { Embed } \\
\text { Time } \\
(\text { Sec })\end{array}$ & $\begin{array}{c}\text { Extractio } \\
\text { n Time } \\
(\text { Sec })\end{array}$ & $\begin{array}{c}\text { Embed } \\
\text { Time } \\
(\text { Sec })\end{array}$ & $\begin{array}{c}\text { Extractio } \\
\text { n Time } \\
(\text { Sec })\end{array}$ \\
\hline & \multicolumn{2}{|c|}{ Lena } & \multicolumn{2}{|c|}{ Baboon } \\
\hline $\begin{array}{c}\text { Yu et } \\
\text { al. [19] }\end{array}$ & N.A. & 248 & N.A. & $\begin{array}{c}\text { More than } \\
10 \text { hrs }\end{array}$ \\
\hline
\end{tabular}

\begin{tabular}{|c|c|c|c|c|}
\hline $\begin{array}{c}\text { Yen et } \\
\text { al. [17] }\end{array}$ & 6 & 2 & 72 & 42 \\
\hline $\begin{array}{c}\text { Agarw } \\
\text { al et } \\
\text { al. [10] }\end{array}$ & 16.37 & 15.20 & 14.23 & 14.04 \\
\hline $\begin{array}{c}\text { Mishra } \\
\text { et al. } \\
\text { [14] }\end{array}$ & 2.65 & .03 & 2.64 & .01 \\
\hline
\end{tabular}

\section{ANALYSIS}

It is clear from this survey of literature that adaptive soft computing techniques are being extensively used to carry out image watermarking both in grayscale and colored images. As stated in the introduction that presently image watermarking is perceived as an optimization problem which requires the usage of these techniques to enable it to strike a balance between visual quality of the signed images and the robustness of the embedding scheme. In particular, neural networks are traditionally considered as adaptive formulations which are able to do so. However, due to the involvement of inherent mathematics according to the selected formulation, the performance differs from one model to another. In general, BPN based networks are successful in optimizing the twin parameters, yet they use to get trapped in local minima and it is quite possible that they do not at all find a global optimal solution to this problem. This leads to consumption of a large training time span in this case. However, it is very clear that if a BPN is successfully trained, it resolves this issue to certainty. The Radial basis function neural networks are indeed free from these defects and attempt to resolve this issue in an iterative manner. However, the time span to train a RBF-NN on a reasonably large host image ranges from 15-22 seconds. Moreover, the RBF-NN is implemented in DWT domain to carry out image watermarking which ensures that the visual quality of the signed images is better in comparison to DCT based BPN approach. This clearly makes it a good candidate for implementing grayscale image watermarking. Hybrid approaches which are primarily adaptive in nature are also presented. These include a GA-BPN based approach. The results of this approach are quite interesting. This algorithm is clearly able to optimize the visual quality and robustness for all four images used in the simulation. Both these parameters are well optimized and the signed images are of good visual quality. Particularly, the algorithm is able to extract the watermarks as indicated by high computed values of the normalized correlation parameter. Although, this paper discusses about time spans consumed in various processes and is a generalized approach, the time spans vary from $14-16$ seconds for a single image. This makes it equally suitable to carry out image watermarking on an individual scale. The Fuzzy Logic-Tabu Search based hybrid approach does give good results in terms of the visual quality and robustness, but it does not give any computation of the time spans for the embedding and extraction processes.

On the contrary, the Extreme Learning Machine based grayscale image watermarking schemes present a comprehensive picture. This algorithm was primarily designed to avoid getting trapped into local minima by the BPN. In this case, the weight selection is done randomly and only a single turing parameter is used. This makes it a fast algorithm to train even a large data set such as that of images. However, several researchers have argued that this does not behave like a generalized algorithm and due to this reason, it 
is possible to obtain a different training and test accuracy for the same train and test data set applied to it in different iterations. However, the visual quality of the images watermarked by using this technique is very good. The PSNR value obtained is in the range of $55-60 \mathrm{~dB}$. The similarity correlation parameter is also very high (18-25) for different images. Moreover, the time spans consumed in various processes is far less than its previous counterparts. The training time span is in milliseconds. The embedding and extraction time spans is in seconds. This makes it a fit candidate to carry out grayscale image watermarking on a real time scale. This is also suitable to develop fast watermarking applications. As the time spans are in milliseconds, it can also be subsequently used to implement watermark embedding and extraction in uncompressed video. The issue of lack of generalization capability may be resolved by implementing suitable changes within the mathematical formulations of this algorithm. One such modification has been carried out and a new variant of the ELM, commonly known as Online sequential ELM (OS-ELM) has been developed. This is widely believed to resolve the problem of lack of generalization capability of the ELM algorithm. Its training and testing accuracies are better and more stable as well. It shall be a good endeavor to develop a fast and stable watermarking application using OS-ELM in the future and to study its characteristics especially to optimize the required parameters.

\section{CONCLUSIONS}

In this paper, a survey of the adaptive soft computing techniques is presented to carry out grayscale image watermarking. A comprehensive set of papers which are published in this direction have been studied and a comparative analysis is presented. It is concluded that although the BPN based watermarking techniques are able to optimize the visual quality and robustness of the embedding scheme, yet it consumes a large time span to finish various processes involved. The Radial basis Function based network is capable to resolve the problem encountered by the BPN based schemes. It is generally implemented in the DWT domain and therefore gives better results in terms of the visual quality of signed images as compared to DCT - BPN based approach. Hybrid approaches are also found suitable for this purpose. GA-BPN based watermarking scheme is quite suitable as the visual quality and robustness issue is optimized by using it. The time spans in different processes are also suitable to carry out embedding and extraction in individual images. It may not be found suitable for implement video watermarking which involves a large number of frames run at a high speed. The ELM based approach is found very suitable both in terms of the optimization of the twin parameters as well as the time computation. The processing time is very less (milliseconds) and this makes it suitable to develop watermarking applications on a real time scale. Precisely due to this reason, in future, it may also be used in video watermarking.

\section{REFERENCES}

[1] P. Meerwald and A. Uhl, A survey on wavelet domain watermarking algorithms, In Proceedings of SPIE, electronic imaging, security and watermarking of multimedia contents III (2001), (Vol. 4314), 505-51

[2] N Nikolaidis and I Pitas, Robust image watermarking in the spatial domain, Signal Process (1998), 66(3), 385403

[3] J. Hernandez, M. Amado and F. Perez-Gonzalez, DCTdomain watermarking techniques for still images:
Detector performance analysis and a new structure, IEEE Transaction on Image Processing (2000), 9(1), 55-67

[4] Saraju P. Mohanty, K.R. Ramakrishnan, Mohan Kankanhalli, A dual watermarking technique for images, ACM Multimedia 2 (1999), pp. 49-51

[5] Song Huang, Wei Zhang, Wei Feng, Huaqian Yang, Blind watermarking scheme based on neural network, Seventh World Congress on Intelligent Control and Automation (WCICA 2008), 2008, pp. 5985-5989

[6] Chuan-Yu Chang and Sheng-Jyun Su, A NeuralNetwork-Based Robust Watermarking Scheme, Proceedings of the IEEE International Conference on Systems, Man and Cybernetics (2005) (Volume 3), 10-12 Oct, 2005, pp 2482-2487

[7] Cheng-Ri Piao, Seunghwa Beack, Dong-Min Woo, and Seung-Soo Han, A Blind Watermarking Algorithm Based on HVS and RBF Neural Network for Digital Image, ICNC 2006, Part I, LNCS 4221, (2006), pp. 493 496

[8] Zhang Zhi-Ming, Li Rong-Yan and Wang Lei, Adaptive watermark Scheme with RBF Networks, Proceedings of the IEEE International Conference on Neural Networks and Signal Processing (2003) (Volume 2), 14 - 17 Dec. 2003, pp. 1517 - 1520

[9] Ali Mohammad Latif, An Adaptive Digital Image Watermarking Scheme using Fuzzy Logic and Tabu Search, Journal of Information Hiding and Multimedia Signal Processing (2013), vol 4, pp. 250-271

[10] Charu Agarwal, Anurag Mishra and Arpita Sharma, Gray-Scale watermarking using GA-BPN Hybrid Network, Journal of Visual Communication and Image Representation, (2013), Vol 24, pp. 1135 - 1146

[11] G-B Huang, Q-Y Zhu and C K Siew, Extreme Learning Machine: Theory and Applications, (2006), Neurocomputing, vol (70), pp 489-501

[12] G-B Huang, Q-Y Zhu and C K Siew, Real-Time Learning Capability of Neural Networks, (2006), IEEE Transactions on Neural Networks, vol 17(4), pp 863-878

[13] G-B Huang (2004), The code for ELM is available on: http://www.ntu.edu.sg/home/egbhuang

[14] Anurag Mishra, Amita Goel, Rampal Singh, Girija Chetty and Lavneet Singh, A Novel Image Watermarking Scheme Using Extreme Learning Machine, Proceedings of IEEE World Congress on Computational Intelligence (WCCI 2012), Brisbane, Australia, June 10-15, 2012, pp 1-6

[15] Ingemar Cox, J. Kilian, F. T. Leighton and T, Shamoon, Secure spread spectrum watermarking for multimedia, IEEE Transaction on Image Processing, (1997), vol. 6, 1673-1687

[16] Rampal Singh, Neelam Dabas, Vikash Chaudhry and Anurag Mishra, On Extreme Learning Machine for Watermarking of Images in Discrete Wavelet Transform Domain, In Proceedings of IEEE $10^{\text {th }}$ International Conference on Intelligent Information Hiding and Multimedia Signal Processing (IIH-MSP 2014), 27-29 August, 2014, Kitakyushu, Japan

[17] Shwu-Huey Yen and Chia-Jen Wang, SVM Based Watermarking Technique, Tamkang Journal of Science and Engineering (2006), Vol. 9, No. 2, pp 141-150

[18] Bibi Isac and V. Santhi, A study on Digital Image and Video Watermarking using Neural Networks, International Journal of Computer Applications, Vol. 12, No. 9, Jan 2011

[19] P. Yu, T. Tsai, H. H and Sun D. W, Digital Watermarking of Color Images using Support Vector Machines, National Computer Symposium (2003) 